\title{
Measuring the impact of contraceptive use on unintended pregnancy and other health outcomes
}

STEP UP Research Programme Consortium

Follow this and additional works at: https://knowledgecommons.popcouncil.org/departments_sbsr-rh

Part of the Demography, Population, and Ecology Commons, Family, Life Course, and Society Commons, International Public Health Commons, Maternal and Child Health Commons, Obstetrics and Gynecology Commons, and the Women's Health Commons How does access to this work benefit you? Let us know!

\section{Recommended Citation}

"Measuring the impact of contraceptive use on unintended pregnancy and other health outcomes," STEP UP Brief. New York: Population Council. 


\section{MEASURING THE IMPACT OF CONTRACEPTIVE USE ON UNINTENDED PREGNANCY AND OTHER HEALTH OUTCOMES}

National and global support for services that enable women and their partners to have access to a full range of quality contraceptives are growing rapidly, as evidenced through the financial commitments made during the London Summit on Family Planning, and the ensuing FP2020 Initiative that seeks to reduce unmet need for such services by 120 million women.

With these investments, and the anticipated increases in effective contraceptive use, come expectations of substantial and demonstrable impacts on a range of health outcomes, including significant reductions in unintended pregnancies, maternal, newborn and child deaths and unsafe abortions.

Attributing health outcomes to a specific health intervention is always challenging, and direct measurement of outcomes averted is not possible. Consequently, several statistical models have been developed that estimate the number of health outcomes that will be averted because of contraceptive use. The table on page 3 describes and compares five of the most commonly used approaches: Adding it Up (AIU); Impact2; ImpactNow; Reality Check; and FamPlan/LiST. AIU is used by staff at the Guttmacher Institute for undertaking and presenting analyses of outcomes and is not in the public domain; the other four estimation models have been developed for use at national and sub-national levels by donors, programme managers and policymakers. While these are the most frequently used approaches to measure the impact of contraceptive use, other models are available that address similar

\section{APPROACHES FOR MEASURING THE IMPACT OF CONTRACEPTIVE USE}

\section{Adding it up (AIU)}

ORGANISATION: Guttmacher Institute CONTACT: Jacqueline E. Darroch (JEDarroch@guttmacher.org)

WEBSITE: Darroch, J, Singh S. 2011. Adding It Up: The Costs And Benefits Of Investing In Family Planning And Maternal And Newborn Health-Estimation Methodology. New York: Guttmacher Institute. www.guttmacher.org/pubs/AIU-methodology.pdf

\section{Impact2}

ORGANISATION: Marie Stopes International

CONTACT: Michelle Weinberger (michelle.weinberger@mariestopes.org)

WEBSITE: Marie Stopes International. 2012. Impact 2: An innovative tool for measuring the impact of reproductive health programmes. London: Marie Stopes International. www.mariestopes.org/impact-2

\section{ImpactNow}

ORGANISATION: USAID Health Policy Project, Futures Group

CONTACT: Ellen Smith (esmith@futuresgroup.com)

WEBSITE: Not yet available

\section{Reality Check}

ORGANISATION: USAID RESPOND Project, EngenderHealth CONTACT: Melanie Yahner

(myahner@engenderhealth.org)

WEBSITE: Not yet available www.respond-project.org

\section{FamPlan/LiST}

ORGANISATION: USAID Health Policy Project, Futures Institute

CONTACT: John Stover

(jstover@futuresinstitute.org)

WEBSITE: Spectrum Manual: Spectrum System of Policy Models: www.futuresinstitute.org/spectrum.aspx

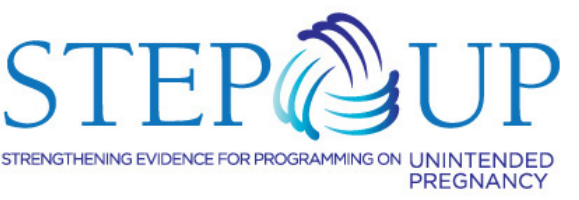


questions. The Crosswalk document ${ }^{1}$ helps advocates, programme planners, decision makers, and others select which among these tools might best address their goals or questions, as well as provide insight into how to interpret the outputs of each tool.

However, concerns have been expressed that this range of approaches-which overlap in the impacts they estimate, but sometimes use different inputs, mathematical algorithms and assumptions, and may produce different results-may be confusing for policy makers, managers and donors who want to measure or evaluate these impacts. To address these concerns, the STEP UP consortium convened two expert meetings (in September 2013 and March 2014) to review the estimation methods used; come to consensus on methodologies and assumptions, where possible; and provide guidance to those wanting to measure such impacts. Following the first meeting, the same data from four countries (Bangladesh, Burkina Faso, Colombia, Malawi) were inputted into each model and the estimates generated for numerous impacts were compared. Substantial differences emerged, which provided the basis for intensive discussions between the model developers during the second meeting. These discussions led to modifications in the models through reaching a consensus on the following core elements.

\section{PREGNANCY RATES}

Pregnancy rates are important for determining the likelihood of a pregnancy among sexually active, fecund women who do not want to become pregnant when contraception is not used. Agreement was reached that the same pregnancy rate for a "non-user at risk of unintended pregnancy" should be used as the default where applicable in all models and kept constant for the foreseeable future. Using the AIU methodology, the estimated pregnancy rate among women with unmet need using no method across 148 developing countries is $31 \%$, with an inter-quartile plausibility range of $23-38 \%$. This rate is calculated by dividing the number of unintended pregnancies among the estimated number of women with unmet need using no method, by the total number of women with unmet need using no method. This rate is lower than some previous estimates. It should be noted that this default pregnancy rate will be adopted by the models Impact2, ImpactNow, Reality Check and FamPlan but the published tables in Adding It Up will continue to use Guttmacher's estimates of regional pregnancy rates.

\section{CONTRACEPTIVE METHOD FAILURE RATES}

Agreement was reached that the same method failure rates should be used where applicable in all models because it is important to ensure that the estimated impact of using contraception takes into account the fact that no method is $100 \%$ effective. The group recommended using the method-specific rates calculated by Cleland, Ali \& Shah ${ }^{1}$ and by Trussell. ${ }^{2}$ Moreover, agreement was reached that these rates should not be adjusted for individual countries because: $i$ ) it is not possible to distinguish unintended pregnancy by method used in national settings; and ii) these global estimates are widely accepted. As with the pregnancy rates, this consensus will be implemented by the models Impact 2, ImpactNow, Reality Check and FamPlan; the Adding It Up analysis will continue to be based on the adjusted failure rates estimated by Guttmacher staff, which differ by region.

\section{ESTIMATING ABORTIONS AND MISCARRIAGES AVERTED}

AIU, Reality Check, and FamPlan will use the "\% of unintended pregnancies ending in abortion" rates estimated by the Guttmacher Institute. Impact2 and ImpactNow currently apply sub-regional abortion ratios to the number of live births averted, and will explore using the Guttmacher Institute's unintended pregnancy ratios instead. The models estimate the number of miscarriages averted slightly differently, but the differences are very minor and so no change was deemed necessary.

\section{MATERNAL DEATHS AVERTED}

Published national maternal mortality ratios (MMR) represent the overall risk of maternal mortality given the national distribution of pregnancy outcomes (births, abortions, miscarriages). Applying these ratios to estimates of live births averted by contraceptive use does not accurately represent the risk of maternal mortality of an adjusted distribution on unintended pregnancies (see above). Reality Check and FamPlan will adapt the AIU approach to adjusting the national maternal mortality ratio estimations, ${ }^{4}$ to reflect the adjusted distribution of unintended pregnancy outcomes. This adjustment will be done using WHO sub-regional unsafe abortion rates and a Guttmacher Institute estimate of two deaths per 100,000 safe abortions. Impact2 and ImpactNow will explore using this adjusted approach as part of exploring adjusting pregnancy outcomes based on pregnancy intention. 


\section{Adding It Up \\ Guttmacher Institute \\ Illustrate the health and} financial costs and benefits of modern contraceptive use in terms of outcomes averted through current or full (i.e. no unmet need) contraceptive use.

Apply current proportion of sub-regional unintended pregnancies that end in abortions (safe \& unsafe) to unintended pregnancies averted.

\section{Apply mortality ratios for live} births, safe \& unsafe abortions averted; Ratios based on WHO country MMR, WHO regional unsafe abortion mortality and estimated safe abortion mortality.

\section{Attribute infant mortality} reduction to fewer births.

Apply WHO or IHME mortality rates to unplanned births averted.
Estimate the current and potential future impacts of FP service provision on adverse outcomes averted through comparing current contraceptive use (modelled from service statistics) with no use;

Estimate the quantity of contraceptive services needed to reach a specified contraceptive prevalence goal.

Quantity of services provided annually; or programme goal (CPR, additional users)

The following inputs have defaults which can be overridden by the user:

- Demographic data from UN Population Projections;

DHS and UN Population Division data on CPR, unmet need $\%$ married;

- Special studies for method effectiveness and CYP factors;

- Costs \& coverage by of maternal \& neonatal interventions from UN Reproductive Health Costing Tool

\section{Programme outcomes:}

- FP users by method

- Contribution to CPR

- Services needed to reach goa

Impacts averted:

- Unintended pregnancies;

- Live births;

- Safe \& unsafe abortions;

- Maternal \& child deaths;

- DALYs;

- Direct costs saved to families and healthcare systems (maternal \& neonatal).

\section{Required input from user:} given FP budget.

Apply sub-regional abortion ratios to the number of live births averted nationally; Live births averted calculated by subtracting estimated abortions, stillbirths, and miscarriages from pregnancies averted.

Apply the national maternal mortality ratio to the number of live births averted Default MMR values from WHO provided.

Country-specific coefficient applied to the number of live births; this coefficient estimates the effect of longer birth intervals on decreasing child mortality.

Apply coefficient developed using PSI methodology for birth spacing impact. births averted calculated by mortality ratio to the number of
Estimate the health and economic impacts of contraceptive use in the near-term; automatic scenario comparisons facilitate creation of advocacy messages. Can also estimate impacts for a

The following inputs have default values, which can be overridden by the user:

- Demographic data from UN Population Projections;

- DHS and UN Population

Division data on CPR, unmet need, \% Married;

- Special studies for method effectiveness and discontinuation;

- Costs \& coverage by of maternal \& neonatal interventions from UN Reproductive Health Costing Tool and others;

- Unit costs of FP provision (by method).

\section{Programme outcomes:}

FP users and acceptors, by method

- CPR;

- Unmet need;

- FP budget required (depending on configuration chosen)

\section{Impacts averted:}

- Unintended pregnancies:

\section{- Live births;}

- Safe \& unsafe abortions;

- Maternal \& child deaths;

- DALYs;

- Maternal \& neonatal costs averted;

- Cost-benefit ratio:

- ICER (Incremental CostEffectiveness Ratio).

Estimate the resources required to maintain or reach a specified contraceptive prevalence, increase by a certain annual rate, or meet unmet need;

Estimate the potential impact of achieving this goal on averting adverse outcomes.

The following inputs have default values which can be overridden by the user:

- Method discontinuation, CYP factors, per-unit costs, pregnancy rate, unintended pregnancy outcomes, maternal mortality ratio;

- Tabulations of most recent national surveys (e.g. DHS MICS) for CPR

- Demographic data from UN Population Projections.

Programme outcomes:

- FP users and acceptors;

- Commodities

Commodity costs

Impacts averted:

- Unintended pregnancies:

- Live births;

- Safe \& unsafe abortions;

- Maternal deaths.

Apply sub-regional abortion ratios to the number of live births averted nationally; Live subtracting estimated abortions, stillbirths, and miscarriages from pregnancies averted.

Apply the national maternal live births averted. Default MMR values from WHO provided.

\section{Country-specific coefficient} applied to the number of live births; this coefficient estimates the effect of longer birth intervals on decreasing child mortality.

Apply coefficient developed using PSI methodology for birth spacing impact.

Apply current proportion of sub-regional unintended pregnancies that end in abortions (safe \& unsafe) to unintended pregnancies averted.

Apply MMR to number of live births averted. Default MMR values from WHO provided.
Infant and child deaths averted are not outputs of the tool.

Futures Institute

Estimate the resources required to meet contraceptive goals, such as reducing unmet need, achieving a specified modern contraceptive prevalence gaol, or achieving a goal of reduced total fertility.

The following inputs have default values preloaded, which cannot be overridden by the user:

- Demographic data on population by age and sex in base year, TFR, age distribution of fertility, sex ratio at birth, life expectancy at birth, migration by age and sex from UN Population Projections.

- DHS data on method mix, source mix, proximate determinants of fertility, costs of FP by method and source, method effectiveness, CYP factors, and percent of births that are high risk;

- Data from special studies for method effectiveness and CYP factors.

Programme outcomes:

- TFR;

$-\mathrm{CPR}$

- FP users and acceptors, by method and source;

- Unmet need;

- Average method effectiveness: - CYP

- Commodities required;

- Contraceptive service costs

- Percentage of births with demographic risk factors:

- Pregnancies;

- Births by mother's age, birth interval and parity;

- Abortions;

- Maternal deaths, by type

- Infant and under 5 mortality rates

Impacts averted:

- Impacts can be calculated for any of the programme outcomes listed above

Apply MMR to the number of live births.

Number of abortions calculated from an assumed total abortion rate, or as a percentage of unintended pregnancies.

Deaths averted result from two factors: 1) contraception leads to fewer births which result in fewer deaths, 2) contraception leads to fewer risky births (high parity, closely spaced, mother's age $<18$ or $>35$ ) which can reduce mortality rates and thus result in fewer deaths. 


\section{INFANT AND CHILD DEATHS AVERTED}

The estimates of infant and child deaths averted are recognized as the weakest elements of these models because the relationship between contraceptive use and infant and child mortality is still being clarified. However, these impacts are requested by many users for advocacy purposes and so all models are looking to improve the estimation methods; for example, Impact2 and FamPlan are exploring how contraceptive use changes the distribution of birth spacing risks and subsequent child mortality. AIU currently estimates infant deaths averted due to a reduction in the number of births (i.e. the demographic effect), rather than changes in risk and will explore integrating findings regarding changes in birth spacing.

\section{IMPLICATIONS FOR MEASURING THE IMPACT OF CONTRACEPTIVE USE}

These meetings and modelling exercises provided an important and unique opportunity for those involved in the development of estimation methodologies to come together and to discuss the various approaches used. This review process enabled the group to identify where and why differences existed and to seek alignment for harmonising the modelling approaches more closely. As a result, the estimation methodologies for each approach have been coordinated and consensus reached to ensure that, wherever possible, they are not in conflict. This alignment and consensus building process has strengthened the models by enabling their developers to benefit from each other's' experience and research. Moreover, decision-makers and managers using the different models can more clearly understand the assumptions behind each model so as to make informed choices between them.

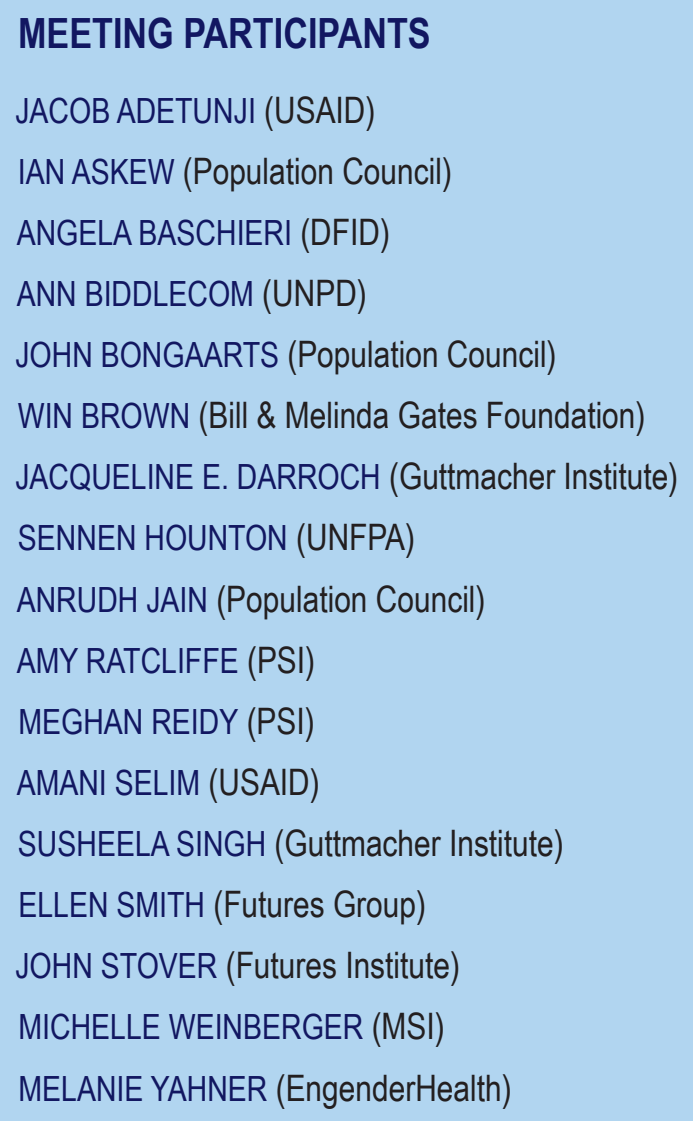

STEP UP generates policy-relevant research to promote an evidence-based approach for improving access to family planning and safe abortion services.

We work in Bangladesh, northern India, Ghana, Kenya, and Senegal.
Population Council Coordinating Partner

African Population and Health Research Center icddr,b
London School of Hygiene and Tropical Medicine

Marie Stopes International

Partners in Population and Development

Funded by

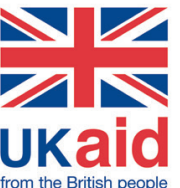

